\title{
Association analysis identifies ZNF750 regulatory variants in psoriasis
}

\author{
Ramon Y Birnbaum ${ }^{1,2,3 \dagger}$, Genki Hayashi ${ }^{4 \dagger}$, Idan Cohen ${ }^{3}$, Annie Poon ${ }^{2,5}$, Haoyan Chen ${ }^{4}$, Ernest T Lam ${ }^{2,5}$, \\ Pui-Yan Kwok ${ }^{2,5}$, Ohad S Birk ${ }^{3}$ and Wilson Liao ${ }^{4^{*}}$
}

\begin{abstract}
Background: Mutations in the ZNF750 promoter and coding regions have been previously associated with Mendelian forms of psoriasis and psoriasiform dermatitis. ZNF750 encodes a putative zinc finger transcription factor that is highly expressed in keratinocytes and represents a candidate psoriasis gene.

Methods: We examined whether ZNF750 variants were associated with psoriasis in a large case-control population. We sequenced the promoter and exon regions of ZNF750 in 716 Caucasian psoriasis cases and 397 Caucasian controls.

Results: We identified a total of 47 variants, including 38 rare variants of which 35 were novel. Association testing identified two ZNF750 haplotypes associated with psoriasis $(p<0.05)$. We also identified an excess of rare promoter and $5^{\prime}$ untranslated region (UTR) variants in psoriasis cases compared to controls ( $p=0.041$ ), whereas there was no significant difference in the number of rare coding and rare $3^{\prime}$ UTR variants. Using a promoter functional assay in stimulated human primary keratinocytes, we showed that four ZNF750 promoter and 5' UTR variants displayed a $35-55 \%$ reduction of ZNF750 promoter activity, consistent with the promoter activity reduction seen in a Mendelian psoriasis family with a ZNF750 promoter variant. However, the rare promoter and 5' UTR variants identified in this study did not strictly segregate with the psoriasis phenotype within families.
\end{abstract}

Conclusions: Two haplotypes of ZNF750 and rare 5' regulatory variants of ZNF750 were found to be associated with psoriasis. These rare $5^{\prime}$ regulatory variants, though not causal, might serve as a genetic modifier of psoriasis.

\section{Background}

Psoriasis is a chronic, inflammatory disorder of the skin affecting $2-4 \%$ of the Caucasian population [1]. Clinically, psoriasis is characterized by red, scaly plaques typically favoring the elbows, knees, scalp, umbilicus, and gluteal cleft and may be associated with nail dystrophy and arthritis. Histologically, psoriasis is marked by epidermal hyperplasia, dilated vasculature in the dermal papillae, and the presence of $\mathrm{T}$ cell infiltrates. Genomewide association studies and other genetic investigations of psoriasis have identified at least 18 common variants affecting psoriasis susceptibility [2-9]. However, in aggregate, these common variants only explain a fraction of the heritability in psoriasis [10]. Therefore, the

\footnotetext{
* Correspondence: liaowi@derm.ucsf.edu

† Contributed equally

${ }^{4}$ Department of Dermatology, University of California, San Francisco,

California, USA

Full list of author information is available at the end of the article
}

missing heritability in psoriasis might be explained by other types of variants not captured by the previous genetic studies, such as rare variants with a low minor allele frequency in the general population.

One candidate susceptibility gene for psoriasis is ZNF750, a gene located at chromosome 17q25 within the PSORS2 locus. ZNF750 has previously been reported to be associated with autosomal dominant forms of psoriasis or psoriasiform dermatitis in two separate, multigenerational families. The first family was a fivegeneration Jewish Israeli family of Moroccan descent in which affected members displayed clinical features of both psoriasis and seborrheic dermatitis [11]. The causative mutation was identified as a ZNF750 frameshift mutation at residue 19 of this 723 -residue protein, resulting in a 44-residue truncated protein which abrogated the zinc finger binding domain. The second family was a five-generation Chinese family with psoriasis [12]. Gene mapping and functional studies identified the
C Biomed Central

() 2011 Birnbaum et al; licensee BioMed Central Ltd. This is an Open Access article distributed under the terms of the Creative Commons Attribution License (http://creativecommons.org/licenses/by/2.0), which permits unrestricted use, distribution, and reproduction in any medium, provided the original work is properly cited. 
most likely causal mutation as a c. $-625 \mathrm{~A}>\mathrm{C}$ promoter variant in ZNF750 that resulted in $42 \%$ reduction in promoter activity.

Since Mendelian-pattern psoriasis in these two large families is associated with ZNF750, we sought to examine whether variants in ZNF750 might influence the development of psoriasis in a larger population. In this study, we investigated whether variants within the ZNF750 promoter, 5' UTR, coding regions, and 3' UTR were associated with psoriasis in a Caucasian population.

\section{Methods}

Patients

DNA samples from 716 unrelated Caucasian psoriasis cases, 397 Caucasian healthy adult controls, and 20 additional family members were collected from the University of California, San Francisco and Washington University, St. Louis. Cases were recruited from outpatient dermatology clinics and the diagnosis of psoriasis was confirmed by a board-certified dermatologist. Healthy controls, recruited from the local community, reported no history of autoimmune disease or cancer according to a written screening questionnaire. All subjects gave written informed consent for study participation in accordance with the institutional review board at their respective institutions.

\section{Sequencing}

Each DNA sample was sequenced over 6 amplicons to cover the promoter and 3 exons of ZNF750. PCR was performed using the following primer pairs, sequences listed 5' to 3': amplicon 1 (531 bp, forward AATACTG TGCCTCCCAGGGTAT, reverse GTACTTACCAGA GGTGGGCAGTG); amplicon 2 (710 bp, forward TGT CCTGACACCAAGACTGC, reverse CGACTGGAAC AAATGCAGAA); amplicon 3 (698 bp, forward GGCAT CACCCTGCAAGAG, reverse GGTTTAACCTGGAAG GACTCG); amplicon 4 (755 bp, forward CAGGCCA GAGTCTGCATTTT, reverse TGGCTGCCAGGTTTA TCTCT); amplicon 5 (673 bp, forward CCCTCAAC CTCTCCAAGAAA, reverse GTGGCCGTAGCTCTGTG AAC); amplicon 6 (783 bp, forward CTAACGCCGGG TTCACAC, reverse GCACCCGTTCACAGGTTAAT). PCR was performed using $8 \mathrm{ng}$ genomic DNA, $0.4 \mu \mathrm{M}$ each forward and reverse primer, $1 \mathrm{x}$ buffer, $4 \mathrm{mM}$ dNTPs, and 0.3U Qiagen (Valencia, CA USA) HotStar Taq and $1 \mathrm{x}$ Q Solution in a $10 \mu \mathrm{L}$ reaction. PCR was cleaned up by incubation with 1x SAP PCR Clean-Up Reagent (PerkinElmer Life Sciences, Inc. Waltham, MA USA) at $37^{\circ} \mathrm{C}$ for one hour. Sequencing reactions contained $2.5 \mu \mathrm{L}$ of clean PCR product, $0.375 \mu \mathrm{M}$ primer and $8.3 \%$ Applied Biosystems (ABI; Foster City, CA USA) BigDye Terminator v3.1 in a $12 \mu \mathrm{L}$ reaction.
Excess dye terminator removal was performed with genCLEAN (Genetix; New Milton, Hampshire, United Kingdom) plates following manufacturer's instructions before sequencing on an ABI 3730xL DNA Analyzer. Sequencing was performed in one direction except for amplicon 6 in which the presence of multiple indels required the use of bidirectional sequencing, using the internal reverse primer AGCCTCTTGATGTTTG TGTGTT and internal forward primer TGGTTGTAAA AACACCTGAATGA. All rare promoter and 5' UTR variants were sequenced a second time in the forward and reverse directions to confirm their accuracy.

\section{Statistical Methods}

Sequencing traces were analyzed with Sequencher (Gene Codes; Ann Arbor, MI USA). Hardy-Weinberg equilibrium p-values were calculated in Haploview to assess sequencing quality and a p-value of 0.05 was used as the significance threshold for exclusion. Individual polymorphisms were tested for association with psoriasis using Fisher's exact test implemented in PLINK. Haploview was used to identify haplotypes using the confidence intervals method and to calculate haplotype associations using a chi-square test. Haploview was also used to perform empirical p-value estimation, using 100,000 permuted experiments. False discovery rate (FDR) q-values were calculated in R. A weighted sum statistic was used to test functionally similar groups of rare variants for association with psoriasis. This approach of combining multiple independent signals has been shown to be significantly more powerful than variant-by-variant or other approaches for the analysis of rare variants [13]. 10,000 permutations were performed to determine significance for each group tested.

\section{5' Rapid Amplification of cDNA Ends (5' RACE)}

SMART RACE cDNA amplification kit (BD Biosciences; Franklin Lakes, NJ USA) was used to perform 5'-RACE of ZNF750 gene. RACE-ready cDNA was synthesized according to the manufacturer's instructions. RACE PCR was performed with gene-specific primers that were used for RT-PCR of ZNF750 (Exon 2, GGAACTCGATCCTGCTCTGA). RACE products were excised from the agarose gel, purified, and cloned into pGEM-T easy vector (Promega Corp; Madison, WI USA). Cloned inserts were sequenced with the T7 and SP6 primers and the DNA sequences obtained were compared to published sequences.

\section{Reporter Constructs for ZNF750 Promoter Assay}

The ZNF750 reference sequence was taken from National Center for Biotechnology Information, with accession number NM_024702. Five candidate mutations in the promoter and 5' UTR region of ZNF750 
were analyzed for changes in luciferase reporter activity. Four novel variants were identified in patients diagnosed with psoriasis and one mutation, c.- $625 \mathrm{~A}>\mathrm{C}$ was identified by Yang et al [12]. An 849 bp insert containing the promoter and 5' UTR (exon 1 portion) of ZNF750 was amplified from human genomic DNA using the primers 5'-CGGCTAGCCAGCAAGCAAGCAGTTTTGGT-3' (NheI) and 5'-CCAAGCTTGGATGTGGCCGGT CTTGGT-3' (HindIII), and cloned into pGL3 Basic vector (Promega) using NheI and HindIII restriction enzymes. QuikChange II XL Site-Directed Mutagenesis Kit (Agilent Technologies) was used to create the 5 variations of interest individually. All vectors were sequenced to verify the location of the variation.

\section{Transfection and Luciferase Assay}

Primary keratinocytes were provided by Dr. Dennis Oh and Dr. Susana Ortiz-Urda (University of California San Francisco, San Francisco, CA). The cells were grown in $500 \mathrm{ml}$ Medium 154CF and Human Keratinocyte Growth Supplement (Invitrogen; Carlsbad, CA USA), and $35 \mu \mathrm{M}$ of $\mathrm{CaCl}_{2}$. A total of $10^{5}$ cells were seeded in each well of a 6-well plate and were transfected after 24 hours with $500 \mathrm{ng}$ of each reporter construct along with $10 \mathrm{ng}$ of pRL-TK vector (Promega) containing the Renilla luciferase gene as an indicator for normalization of transfection efficiency. Transfections were performed using Lipofectamine ${ }^{\mathrm{TM}}$ LTX with PLUS ${ }^{\mathrm{TM}}$ Reagent (Invitrogen), according to the manufacturer's instructions. After 24 hours, the cells were stimulated with $250 \mathrm{ng}$ phorbol-12-myristate-13-acetate (PMA) for the PMA positive set of experiments. Cells were incubated for an additional 24 hours and then analyzed for luciferase activity with the Dual-Luciferase ${ }^{\circledR}$ Reporter Assay System (Promega) and Synergy 2 (BioTek; Winooski, VT USA). Firefly luminescence was normalized to Renilla luminescence and reported as relative luciferase units compared to wild-type sequence. All experiments were performed independently three times using primary keratinocytes from a single donor.

\section{Results}

Identification of ZNF750 Variants in Cases and Controls

To determine whether ZNF750 is associated with psoriasis, we sequenced ZNF750 in 716 Caucasian psoriasis cases and 397 Caucasian controls. We sequenced the ZNF750 promoter region (defined as $400 \mathrm{bp}$ upstream of the transcription start site), 5' UTR, coding regions which include exons 2 and 3, and 3' UTR. In total, we identified 9 common variants (minor allele frequency (MAF) $\geq 2 \%$ in the controls, Table 1) and 38 rare variants (MAF $<2 \%$, Table 2$)$. One polymorphism, a novel 8 bp deletion at position 78381172 (hg18) in the 3' UTR, was out of Hardy-Weinberg equilibrium and was not included. All identified polymorphisms were successfully genotyped in greater than $94 \%$ of samples and the mean genotyping rate per variant was $97.3 \%$. Nearly all variants were single nucleotide variants (SNVs) with the exception of two single base deletions, one located in the 3' UTR (rs71918228) and the other in the 3' downstream region (rs35156590). All of the identified common variants were present in dbSNP Build 133 whereas 35 of 38 (92\%) of the rare variants were novel.

\section{Association Testing of Identified Variants}

To analyze the significance of the identified ZNF750 variations/mutations in psoriasis patients, we first tested each variant alone using a Fisher's exact test. For the common variants (Table 1), only rs35156590 in the immediate 3' downstream region showed a significant association with psoriasis ( $\mathrm{p}=0.028$, OR $0.81[95 \%$ CI 0.68-0.98]). However, adjustment for multiple hypothesis testing using empirical permutation testing of this SNP yielded $p=0.17$. When tested individually, none of the rare variants (Table 2) achieved a significance association with psoriasis (all $\mathrm{p}>0.05$ ).

Table 1 Common variants identified in ZNF750

\begin{tabular}{lllllll}
\hline Name & Position & Property (PolyPhen2 impact) & Alleles & F_case & F_control & Fisher P \\
\hline rs3744165 & 78383731 & Exon 2, 5' UTR & C/A & 0.171 & 0.161 & 0.541 \\
\hline rs12450046 & 78383677 & Exon 2, 5' UTR & C/T & 0.186 & 0.172 & 0.478 \\
\hline rs8074277 & 78382917 & Exon 2, M235V (benign) & A/G & 0.189 & 0.18 & 0.640 \\
\hline rs35653278 & 78382757 & Exon 2, P288L (probably) & C/T & 0.092 & 0.09 & 0.937 \\
\hline rs34188981 & 78382558 & Exon 2, T354T & C/T & 0.021 & 0.021 & 1.000 \\
\hline rs12948179 & 78381781 & Exon 3, P566P & T/C & 0.393 & 0.42 & 0.233 \\
\hline rs12938126 & 78381754 & Exon 3, A575A & A/G & 0.392 & 0.42 & 0.232 \\
\hline rs71918228 & $78381176-79$ & Exon 3, 3' UTR & CAAA/- & 0.465 & 0.478 & 0.586 \\
\hline rs35156590 & 78380584 & 3' Downstream & $-/ T$ & 0.369 & 0.418 & $\mathbf{0 . 0 2 8}$ \\
\hline Com
\end{tabular}

Common variants are defined as those with minor allele frequency (MAF) $\geq 2 \%$ in controls. Position is on Chr17 (hg18). UTR, untranslated region. PolyPhen2 impact given as benign, possibly damaging, or probably damaging. Alleles are given as major/minor on the (-) strand. F_case, MAF in cases; F_control, MAF in controls. 
Table 2 Rare variants identified in ZNF750

\begin{tabular}{|c|c|c|c|c|c|c|}
\hline Name & Position & Property (PolyPhen2 impact) & Alleles & F_case & F_control & Fisher $\mathrm{P}$ \\
\hline Novel_1 (c.-597C>T) & 78391506 & Promoter & $\mathrm{C} / \mathrm{T}$ & 0 of 1312 & 1 of 745 & 0.363 \\
\hline Novel_2 (c.-458G>A) & 78391367 & Promoter & $\mathrm{G} / \mathrm{A}$ & 1 of 1309 & 0 of 746 & 1.000 \\
\hline Novel_3 (c.-261G>A) & 78391170 & Exon $1,5^{\prime}$ UTR & $\mathrm{G} / \mathrm{A}$ & 1 of 1309 & 0 of 746 & 1.000 \\
\hline Novel_4 (c.-233 C>T) & 78391142 & Exon $1,5^{\prime}$ UTR & $\mathrm{C} / \mathrm{T}$ & 4 of 1306 & 0 of 746 & 0.303 \\
\hline Novel_5 (c.-232G>A) & 78391141 & Exon $1,5^{\prime}$ UTR & $\mathrm{G} / \mathrm{A}$ & 1 of 1311 & 0 of 746 & 1.000 \\
\hline Novel_6 (c.-45G>C) & 78383664 & Exon 2, 5' UTR & $\mathrm{G} / \mathrm{C}$ & 1 of 1349 & 0 of 746 & 1.000 \\
\hline Novel_7 (c.-36A>G) & 78383655 & Exon 2, $5^{\prime}$ UTR & $A / G$ & 1 of 1349 & 0 of 746 & 1.000 \\
\hline Novel_8 (c.-32G>A) & 78383651 & Exon 2, 5' UTR & $\mathrm{G} / \mathrm{A}$ & 1 of 1349 & 0 of 746 & 1.000 \\
\hline Novel_9 & 78383521 & Exon 2, T33T & $\mathrm{T} / \mathrm{C}$ & 1 of 1349 & 0 of 746 & 1.000 \\
\hline Novel_10 & 78383438 & Exon 2, R61Q (possibly) & $\mathrm{G} / \mathrm{A}$ & 2 of 1348 & 1 of 745 & 1.000 \\
\hline Novel_11 & 78383402 & Exon 2, P73L (probably) & $\mathrm{C} / \mathrm{T}$ & 0 of 1350 & 1 of 745 & 0.357 \\
\hline Novel_12 & 78383393 & Exon 2, T76l (benign) & $C / T$ & 0 of 1350 & 1 of 745 & 0.357 \\
\hline Novel_13 & 78383325 & Exon 2, D99N (probably) & $\mathrm{G} / \mathrm{A}$ & 1 of 1349 & 1 of 745 & 1.000 \\
\hline Novel_14 & 78383272 & Exon 2, E116E & $\mathrm{G} / \mathrm{A}$ & 1 of 1349 & 0 of 746 & 1.000 \\
\hline Novel_15 & 78383216 & Exon 2, A135E (benign) & $\mathrm{C} / \mathrm{A}$ & 0 of 1350 & 1 of 745 & 0.357 \\
\hline Novel_16 & 78383196 & Exon 2, A142T (possibly) & $\mathrm{G} / \mathrm{A}$ & 1 of 1349 & 0 of 746 & 1.000 \\
\hline Novel_17 & 78382899 & Exon 2, E241Q (probably) & $\mathrm{G} / \mathrm{C}$ & 1 of 1357 & 0 of 752 & 1.000 \\
\hline Novel_18 & 78382882 & Exon 2, F246F & $\mathrm{T} / \mathrm{C}$ & 0 of 1358 & 1 of 751 & 0.356 \\
\hline rs35283702 & 78382791 & Exon 2, G277R (probably) & $\mathrm{G} / \mathrm{A}$ & 15 of 1343 & 7 of 745 & 0.825 \\
\hline Novel_19 & 78382759 & Exon 2, H287Q (possibly) & $\mathrm{C} / \mathrm{G}$ & 1 of 1357 & 0 of 752 & 1.000 \\
\hline Novel_20 & 78382489 & Exon 2, F377L (benign) & $\mathrm{C} / \mathrm{G}$ & 0 of 1374 & 1 of 763 & 0.359 \\
\hline rs34687659 & 78382445 & Exon 2, Q392R (possibly) & $\mathrm{A} / \mathrm{G}$ & 0 of 1374 & 1 of 763 & 0.359 \\
\hline Novel_21 & 78382401 & Exon 2, A407T (probably) & $\mathrm{G} / \mathrm{A}$ & 1 of 1373 & 0 of 764 & 1.000 \\
\hline Novel_22 & 78382375 & Exon 2, P415P & $\mathrm{G} / \mathrm{A}$ & 2 of 1372 & 2 of 762 & 0.622 \\
\hline Novel_23 & 78382326 & Exon 2, D432H (possibly) & $\mathrm{G} / \mathrm{C}$ & 2 of 1372 & 0 of 764 & 0.540 \\
\hline Novel_24 & 78382188 & Exon 2, V478I (benign) & $\mathrm{G} / \mathrm{A}$ & 0 of 1374 & 1 of 763 & 0.359 \\
\hline Novel_25 & 78382160 & Intron 2 & $\mathrm{C} / \mathrm{A}$ & 1 of 1373 & 0 of 764 & 1.000 \\
\hline Novel_26 & 78382155 & Intron 2 & $\mathrm{~A} / \mathrm{C}$ & 1 of 1373 & 0 of 764 & 1.000 \\
\hline Novel_27 & 78382047 & Intron 2, 5 bp from exon 3 & $\mathrm{~T} / \mathrm{C}$ & 1 of 1373 & 0 of 764 & 1.000 \\
\hline rs35792712 & 78382015 & Exon 3, P488P & $T / G$ & 0 of 1374 & 1 of 763 & 0.359 \\
\hline Novel_28 & 78381714 & Exon 3, G589R (benign) & $\mathrm{G} / \mathrm{C}$ & 1 of 1365 & 0 of 766 & 1.000 \\
\hline Novel_29 & 78381508 & Exon 3, A657A & $\mathrm{G} / \mathrm{A}$ & 1 of 1365 & 0 of 766 & 1.000 \\
\hline Novel_30 & 78381034 & Exon $3,3^{\prime}$ UTR & $\mathrm{G} / \mathrm{A}$ & 1 of 1357 & 0 of 756 & 1.000 \\
\hline Novel_31 & 78380851 & Exon 3, 3' UTR & $A / G$ & 1 of 1365 & 2 of 752 & 0.290 \\
\hline Novel_32 & 78380743 & Exon $3,3^{\prime}$ UTR & $A / G$ & 2 of 1364 & 0 of 754 & 0.541 \\
\hline Novel_33 & 78380697 & Exon $3,3^{\prime}$ UTR & $T / G$ & 1 of 1365 & 0 of 754 & 1.000 \\
\hline Novel_34 & 78380590 & 3' Downstream & $\mathrm{T} / \mathrm{C}$ & 2 of 1318 & 1 of 751 & 1.000 \\
\hline Novel_35 & 78380548 & 3' Downstream & $\mathrm{G} / \mathrm{A}$ & 17 of 1305 & 9 of 743 & 1.000 \\
\hline
\end{tabular}

Rare variants are defined as those with minor allele frequency (MAF) $<2 \%$ in controls. SNPs Novel_ 1 through Novel_ 8 are additionally named by the nucleotide position relative to the translation start site with respect to the cDNA reference. Position is on Chr17 (hg18). UTR, untranslated region. PolyPhen2 impact given as benign, possibly damaging, or probably damaging. Alleles are given as major/minor on the (-) strand. F_case, MAF in cases; F_control, MAF in controls.

Next, we evaluated whether common variant haplotypes of ZNF750 were associated with psoriasis. Two 8-marker haplotypes were significantly associated with psoriasis (Table 3), the risk haplotype CCACCCG(-) $($ frequency $=0.019, \mathrm{p}=0.0011$, OR $3.70[1.45-9.46])$ which includes the risk allele of rs35156590, and the protective haplotype CCACCCGT (frequency $=0.374$, $\mathrm{p}=0.0106,0.79$ [0.66-0.94]) which contains the nonrisk allele of rs35156590. Both of these haplotypes remained significant after correction for multiple comparisons using empirical permutation testing $(\mathrm{p}=$ 0.0024 and $\mathrm{p}=0.0311$, respectively) as well as by 
Table 3 Haplotype association testing between cases and controls

\begin{tabular}{lllllll}
\hline Haplotype & Hap Freq & F_case & F_control & P Value & Emp P-Val & Odds Ratio [95\% CI] \\
\hline CCACCCGT & 0.374 & 0.354 & 0.41 & $\mathbf{0 . 0 1 0 6}$ & $\mathbf{0 . 0 3 1 1}$ & $\mathbf{0 . 7 9}[\mathbf{0 . 6 6 - 0 . 9 4 ]}$ \\
\hline CCACCTA(-) & 0.246 & 0.253 & 0.234 & 0.3321 & 0.9359 & - \\
\hline ACACCTA(-) & 0.137 & 0.138 & 0.136 & 0.9158 & 1.0000 & - \\
\hline CTGCCTA(-) & 0.089 & 0.091 & 0.085 & 0.6426 & 0.9995 & - \\
\hline CTGTCTA(-) & 0.089 & 0.091 & 0.086 & 0.7198 & 0.9997 & - \\
\hline ACACTTA(-) & 0.02 & 0.02 & 0.02 & 0.9666 & 1.0000 & - \\
\hline CCACCCG(-) & 0.019 & 0.026 & 0.006 & $\mathbf{0 . 0 0 1 1}$ & $\mathbf{0 . 0 0 2 4}$ & $\mathbf{3 . 7 0 ~ [ 1 . 4 5 - 9 . 4 6 ]}$ \\
\hline
\end{tabular}

The eight marker haplotype, as ordered in the table, consists of: rs3744165, rs12450046, rs8074277, rs35653278, rs34188981, rs12948179, rs12938126, rs35156590. Hap Freq, overall haplotype frequency; F_case, haplotype frequency in cases; F_control, haplotype frequency in controls; Emp P-Val, empiric p-value calculated by permutation testing

calculation of the false discovery rate $(\mathrm{q}=0.0077$ and $\mathrm{q}=0.037$, respectively).

Due to the well-recognized difficulty in assessing the significance of rare variants on a variant-by-variant basis due to power limitations [14], we utilized a weighted sum statistic [13] to evaluate functional groups of rare variants for psoriasis association. We tested the following rare variant groups: 5' regulatory variants (promoter and 5' UTR), all coding variants, non-synonymous variants, predicted deleterious non-synonymous variants, 3' UTR variants, and all rare variants. We found that only the 5 ' regulatory variants trended towards a significant association with psoriasis (10 variants in cases, 1 variant in controls, unadjusted $\mathrm{p}=$ 0.041, Bonferroni threshold $p=0.008$, Table 4). The putative association of the rare 5' regulatory variants with psoriasis was not secondary to their occurrence on a risk haplotype background. Of the 10 patients with rare 5' regulatory variants, only 1 patient was heterozygous for the risk haplotype CCACCCG(-), while 7 patients were actually heterozygous for the protective haplotype CCACCCGT.

\section{Alternative splicing of ZNF750 mRNA}

In order to characterize the ZNF750 promoter and transcripts, the size of ZNF750 mRNA was verified using two methods: reverse transcriptase PCR (RT-PCR) and 5' Rapid Amplification of cDNA Ends (5' RACE). Using RT-PCR, the predicted cDNA segments were amplified from mRNA of normal individual skin biopsies. Comparison of the cDNA sequences to NCBI and UCSC databases indicated that there are no alternative splicing variants. 5' Rapid Amplification of cDNA Ends performed for ZNF750 in primary keratinocytes produced four 5' RACE PCR products that were cloned and sequenced (data not shown). Alignments of the sequences of the 5' RACE PCR products with human genomic DNA and known mRNA sequences indicated that only two of the four products were specific to ZNF750. The short product represents the mRNA isoform A as in Refseq gi13375990; NM_024702.1. The long product, previously demonstrated in cDNA from tongue tumor tissue [GenBank:DA436414][15] represents the mRNA isoform $B$ and includes an additional $500 \mathrm{bp}$ of sequence upstream to exon 1 of mRNA isoform A (Figure 1). The expression levels of the 5'RACE isoforms were studied by RT-PCR with forward primers specific to isoform $A$ and isoform $B$, respectively, and a reverse primer specific to exon 2. RT-PCR analyses of several cell lines (including primary keratinocytes) showed that the most abundant variant of ZNF750 transcript corresponds to isoform A. Analysis of all 3 reading frames indicated that isoform $A$ and $B$ encode an identical protein: the addition of $500 \mathrm{bp}$ upstream in isoform B compared to isoform A does not result in a different protein. Further investigation of the protein size using Western Blot demonstrated that ZNF750 codes for a single protein of $100 \mathrm{KDa}$ (data not shown). Our data indicate that mRNA isoform A of ZNF750 is the predominant form of ZNF750 expressed

Table 4 Groupwise association testing of ZNF750 functional groups with psoriasis

\begin{tabular}{llll}
\hline Rare Variant Group & Total Variants, Cases $(\mathbf{n}=\mathbf{7 1 6})$ & Total Variants, Controls $(\mathbf{n}=\mathbf{3 9 7})$ & P-value, Weighted Sum Statistic \\
\hline${\text { 5' Regulatory (Promoter }+5^{\prime} \text { UTR) }}^{\prime}$ & 10 & 1 & $\mathbf{0 . 0 4 1}$ \\
\hline All Coding & 30 & 19 & 0.575 \\
- Non-synonymous & 25 & 15 & 0.560 \\
$\quad$ - Predicted deleterious & 24 & 11 & 0.314 \\
\hline 3' UTR & 5 & 2 & 0.351 \\
\hline All Rare & 67 & 32 & 0.192 \\
\hline
\end{tabular}




\section{5' regulatory variations Deleterious mutation}

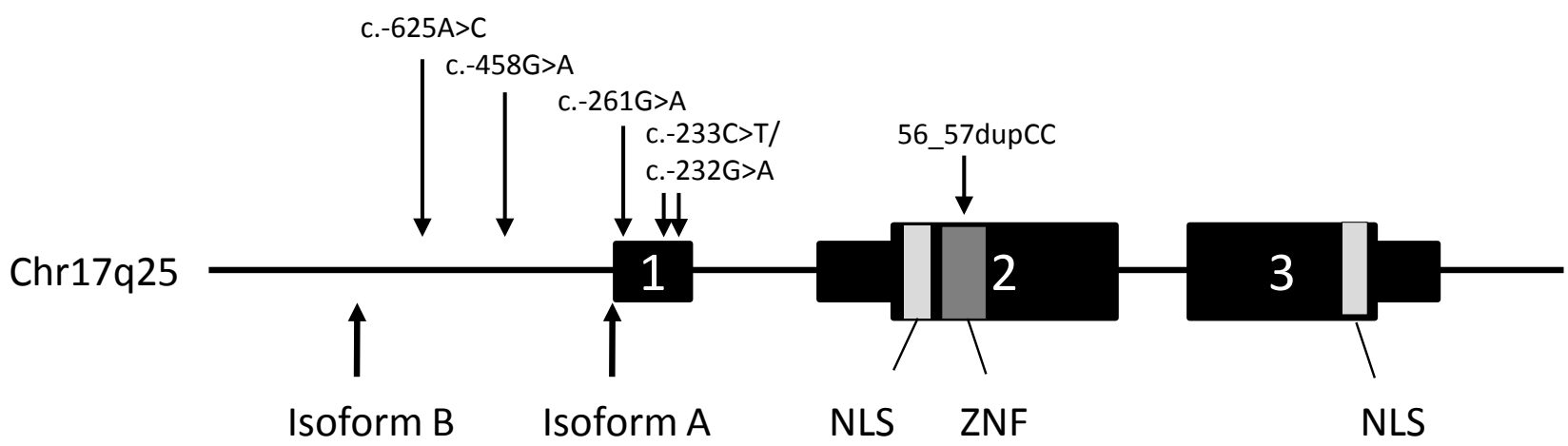

Figure 1 ZNF750 gene structure and variants. ZNF750 consists of 3 exons, with the translation start site within exon 2. Narrow boxes indicate $5^{\prime}$ and $3^{\prime}$ untranslated regions; wider boxes indicate translated regions. ZNF750 contains two nuclear localization sites (NLS) and a C2H2 zinc finger domain (ZNF). Arrows depict the locations of a deleterious frameshift mutation seen in a Jewish Moroccan family (56_57dupCC), promoter and 5' UTR variations examined in this study (c.-625A >C, c.-458G>A, c.-261G>A, c.-233 C>T, c.-232G >A), and start sites of ZNF750 mRNA isoforms $A$ and $B$.

in keratinocytes and that the 5' region DNA variants identified in this study lie within the promoter region and 5' UTR of isoform A.

\section{Functional evaluation of ZNF750 5' Regulatory Variants} To assess whether the ZNF750 5' regulatory variants affect ZNF750 expression levels, we conducted a promoter function assay in human primary keratinocytes. Seven rare variants in the 5 ' regulatory region of ZNF750 were seen in the psoriasis cases and none of these were present in the controls (Table 2). All of these variants were singletons with the exception of c.$233 \mathrm{C}>\mathrm{T}$, which was present in 4 unrelated psoriasis cases. None of these were present in individuals of European descent in the 1000 genomes database. We constructed ZNF750 5' regulatory region fragments with 4 of the variants cloned into individual luciferase reporter assay constructs. The 4 selected variants are located in either the $Z N F 750$ promoter region (c. $-458 \mathrm{G}>\mathrm{A}$ ) or ZNF750 untranslated first exon (c.-261G $>$ A, c. $-233 \mathrm{C}>\mathrm{T}$, c.-232G $>$ A) (Figure 1). Each construct was transfected into human primary keratinocytes which are known to highly express ZNF750 [11]. As an external control, we also tested the activity of the c. $-625 \mathrm{~A}>\mathrm{C}$ variant which was shown to reduce promoter activity in the Taiwanese familial psoriasis study [12]. We found that the c.458G >A promoter variant and the c.-261G $>$ A 5' UTR variant showed a significant reduction of $35 \%$ and $54 \%$ in promoter activity, respectively $(\mathrm{p}<0.001)$. Both c.$233 \mathrm{C}>\mathrm{T}$ and c. $-232 \mathrm{G}>\mathrm{A}$ in the 5' UTR showed a nonsignificant reduction of $13 \%$ in promoter activity (Figure $2 \mathrm{~A})$. Our external control, the c. $-625 \mathrm{~A}>\mathrm{C}$ variant, showed a similar significant reduction of $40 \%$ in promoter activity as previously published [12]. Interestingly, human primary keratinocytes that have been stimulated with phorbol 12-myristate 13-acetate (PMA) have about 10 fold higher ZNF750 promoter activity than unstimulated keratinocytes. Therefore, we tested the effects of the 4 variants on promoter activity after stimulation of human primary keratinocytes with PMA. We found that all 4 of the variants, as well as the external control, showed a significant reduction in promoter activity of $35-55 \%$ compared to the wild type promoter (all $\mathrm{p}<0.001$, Figure 2B).

\section{Clinical Phenotype of Psoriasis Patients with 5' Regulatory Variants}

To assess whether psoriasis patients with 5' regulatory variants have a similar clinical phenotype, we clinically characterized the psoriasis patients with the ZNF750 5 variants (Table 5). Of the 7 affected patients with detailed clinical information available, 5 reported involvement of the scalp or inverse skin folds, and a negative history of psoriatic arthritis, similar to the sebo-psoriasis, arthritis-negative phenotype previously described in the Israeli-Moroccan family with ZNF750 mutation [11].

\section{Discussion}

To our knowledge, this is the first large resequencing study of the ZNF750 gene in psoriasis. As ZNF750 resides in the PSORS2 region [16-19] and has been previously reported to cause familial psoriasis forms of psoriasis [11,12], we were motivated to evaluate ZNF750 in a large cohort of cases and controls. We sequenced a total of 1,113 individuals (716 Caucasian psoriasis cases and 397 ethnically matched healthy controls) in the 


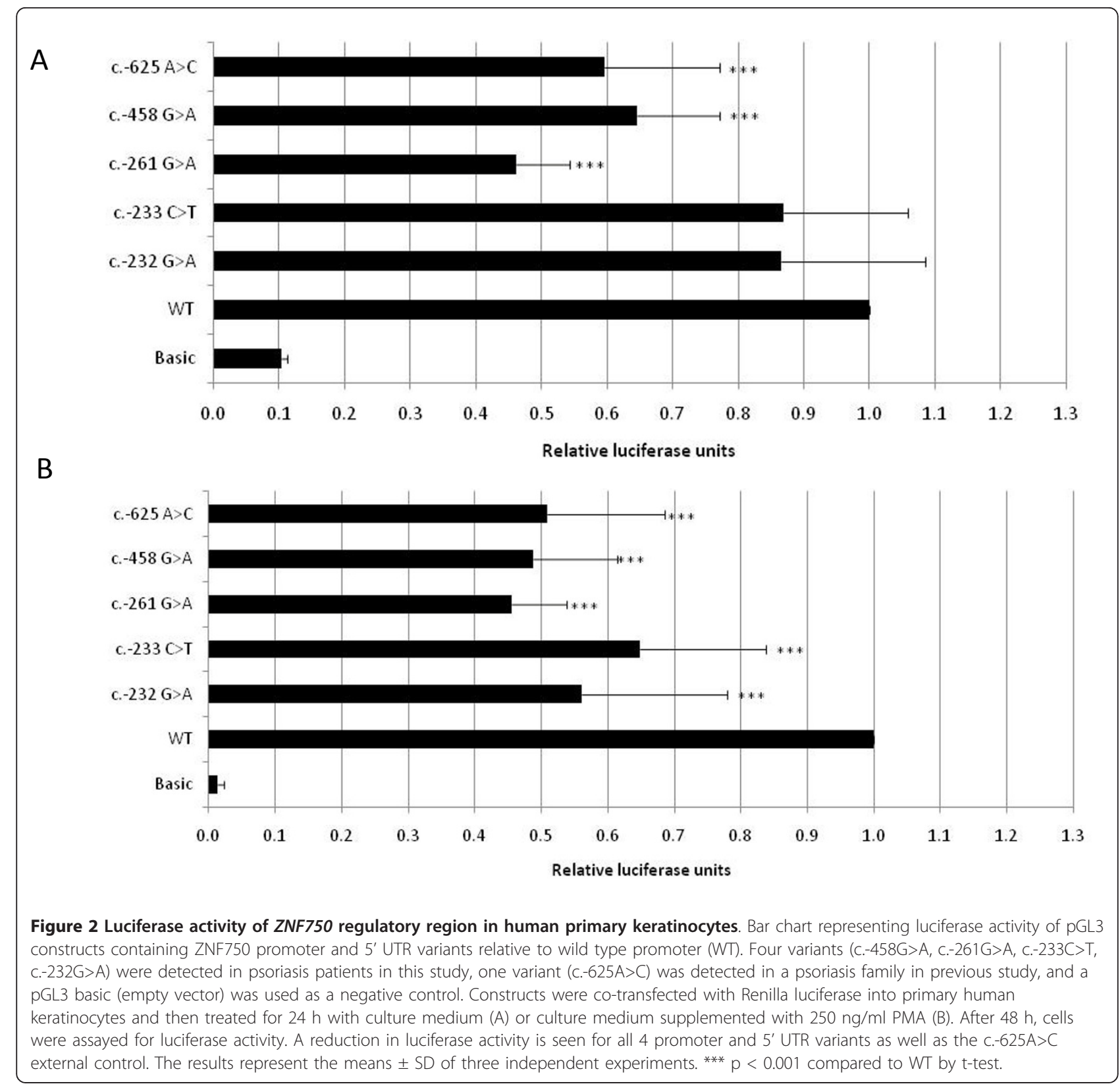

promoter and exonic regions of ZNF750 and identified 47 polymorphisms, of which 35 were novel. According to estimates of Ionita-Laza et al [20], our study captured greater than $99 \%$ of the variants with frequency greater than 0.001 in the CEPH population. However, for perspective, we would have needed to sequence 3,521 individuals to capture $100 \%$ of the variants with frequency greater than 0.001 .

We conducted an association analysis of our identified variants and found no evidence that individual common polymorphisms (MAF > 2\%) were associated with disease. Similarly, imputation of HapMap3/1000 Genomes SNPs using psoriasis GWAS data [7] in the interval 200 $\mathrm{kb}$ upstream to $200 \mathrm{~kb}$ downstream of ZNF750 did not find any SNPs with association p-values less than $1.0 \times$ $10^{-3}$ (data not shown), suggesting that no common ZNF750 SNPs are causal for psoriasis. However, our finding that two ZNF750 haplotypes were significantly associated with psoriasis suggests the possibility that other SNPs on 17q in LD with these haplotypes could possibly be associated with psoriasis. We also detected several rare, non-synonymous, potentially deleterious (PolyPhen2) coding variants in ZNF750 that were only present in cases and not in controls, including A142T, E241Q, H287Q, and A407T (Table 2). However, given that each of these variants was only detected in a single 
Table 5 Clinical characteristics of subjects with rare ZNF750 variants in the $5^{\prime}$ regulatory region

\begin{tabular}{|c|c|c|c|c|c|c|}
\hline $\begin{array}{l}\text { ZNF750 } \\
\text { Variant }\end{array}$ & $\begin{array}{l}\text { \# Pso } \\
\text { Cases }\end{array}$ & $\begin{array}{l}\text { Age: Study } \\
\text { Entry (Onset) }\end{array}$ & Gender & PsA & $\begin{array}{l}\text { Family History of } \\
\text { Psoriasis }\end{array}$ & Description \\
\hline $\begin{array}{l}\text { C.- } \\
458 \mathrm{G}>\mathrm{A}\end{array}$ & 1 & NA & Female & Yes & Sister & Confirmed psoriasis, no other information available \\
\hline C.- $261 \mathrm{G}>\mathrm{A}$ & 1 & $57(35)$ & Male & No & $\begin{array}{l}\text { Mother, brother, } \\
\text { daughter, niece, nephew }\end{array}$ & Confirmed psoriasis \\
\hline \multirow[t]{4}{*}{ c. $-233 C>T$} & 4 & $41(25)$ & Female & No & Mother, sister & $\begin{array}{l}\text { Plaque and inverse psoriasis affecting scalp, trunk, extremities, } \\
\text { breast folds, axillae, and genitals. }\end{array}$ \\
\hline & & $32(15)$ & Female & No & Mother & Plaque psoriasis with $10-20 \%$ BSA affecting scalp, trunk, arms, legs \\
\hline & & $34(5)$ & Male & No & Father, sister & $\begin{array}{l}\text { Guttate psoriasis affecting the scalp, trunk, and arms with 5-10\% } \\
\text { BSA }\end{array}$ \\
\hline & & $54(35)$ & Female & No & Sister & Plaque psoriasis affecting elbows, knees, and legs with $10-20 \%$ BSA \\
\hline c.- $232 \mathrm{G}>\mathrm{A}$ & 1 & $43(30)$ & Male & No & Maternal cousin & $\begin{array}{l}\text { Moderate plaque and inverse psoriasis affecting scalp, elbows, legs, } \\
\text { axillae, genitals, and nails }\end{array}$ \\
\hline c. $-45 \mathrm{G}>\mathrm{C}$ & 1 & $30(5)$ & Female & No & Father & $\begin{array}{l}\text { Moderate guttate (originally plaque) and inverse psoriasis affecting } \\
\text { scalp, arms, legs, trunk, axillae, and genitals }\end{array}$ \\
\hline c. $-36 A>G$ & 1 & $40(25)$ & Female & No & Sister & Moderate psoriasis affecting trunk, legs \\
\hline c. $-32 \mathrm{G}>\mathrm{A}$ & 1 & $65(N A)$ & Male & No & NA & Confirmed psoriasis \\
\hline
\end{tabular}

All psoriasis cases were of Caucasian ethnicity. PsA, psoriatic arthritis diagnosed by rheumatologist; BSA, affected body surface area. NA, information not available.

case and absent additional functional data, it is difficult determine whether these variants were truly deleterious. Therefore, in order to more rigorously evaluate the possible association of rare variants in ZNF750 with psoriasis, we used the weighted-sum approach of Madsen and Browning [13] to evaluate functional groups of rare variants for disease association. This method has been found to be robust compared to other rare variant analysis approaches such as CAST, CMC, Variable Threshold, and Li-Leal $[13,21]$. We found that variants in the promoter or 5' UTR of ZNF750 were enriched in the cases compared to controls (10 variants vs 1 variant, $\mathrm{p}=$ 0.041). Interestingly, the 1 variant found in a control but in none of the cases was c.-597C $>\mathrm{T}$, which was previously reported to be present in 1 of 85 sporadic psoriasis patients screened and 0 of 188 normal controls [12]. In the same study, the promoter variant c.-625A>C was linked to a multigenerational Taiwanese psoriasis family and was reported to reduce ZNF750 promoter activity by $42 \%$ [12]. We therefore investigated the functional impact of 4 of our identified 5 ' regulatory variants in a promoter activity assay. We found that all 4 variants, as well as the c.-625A $>\mathrm{C}$ external control, displayed decreased promoter activity compared to the wild-type promoter. The observed effect on ZNF750 promoter activity was more pronounced in the presence of the stimulator PMA, suggesting that the biological effects of these variants could be dependent on environmental variables such as immune stimulation or response to external cues.

We obtained DNA from the family members of 6 probands with the rare 5' regulatory ZNF750 variants. In a segregation analysis examining the transmission pattern of the rare variants in these six families, we did not identify a clear autosomal dominant pattern (data not shown). Considered together with our functional data, two interpretations could be considered. The first is that certain 5' regulatory variants in ZNF750 could serve as a genetic modifier of the psoriasis phenotype or act as an incremental risk modifier similar to the common susceptibility alleles previously identified [4-8]. It should be noted that the HLA-Cw6 allele, which confers the strongest known risk on psoriasis susceptibility, has a penetrance of only $10 \%$ [22]. The existence of rare variants that influence psoriasis risk but which have moderate, non-Mendelian effect sizes is certainly possible but difficult to prove. Alternatively, it is possible that there is no true association of 5' regulatory variants in ZNF750 with psoriasis and that the nominal association observed is the result of type I error. In this case, the functional effects of these variants on ZNF750 promoter activity demonstrated here may not translate into biological relevance for psoriasis. However, of the 7 patients identified in this study with ZNF750 5' regulatory variants and for whom detailed clinical descriptions were available, 5 reported scalp psoriasis and 3 reported inverse psoriasis (axillae, genitals), which is reminiscent of the sebo-psoriasis phenotype in the previously reported Morrocan family with a frame shift ZNF750 mutation [11]. Variants in the promoter and 5' UTR of ZNF750 which decrease ZNF750 expression might therefore be associated with a seborrheic form of psoriasis.

ZNF750 encodes a 723 amino acid protein that contains two nuclear localization sites (NLS), and two histidines and two cysteines that might serve as zinc binding domains. ZNF750 is a putative member of the $\mathrm{C} 2 \mathrm{H} 2$ 
subclass of zinc finger transcription factors. ZNF750 is highly expressed in keratinocytes, which are the major skin cell type affected both in seborrheic dermatitis and in psoriasis. In addition, human primary keratinocytes that have been differentiated with $\mathrm{Ca}^{2+}$ have increased ZNF750 promoter activity at levels similar to PMA stimulated cells (unpublished data), suggesting ZNF750 may serve an important function in keratinocyte differentiation or immune response in the skin. As keratinocytes in psoriasis or seborrheic dermatitis secrete factors that recruit cells of the immune system and help maintain the inflammatory response [23,24], it is possible that an insufficient level of ZNF750 could lead to a downstream effect that fails to repress a stimulated immune response in psoriasis or seborrheic dermatitis.

\section{Conclusions}

In summary, we have performed the first re-sequencing study of the candidate psoriasis gene ZNF750 in a large case-control population. Although no individual variants were found to associate with psoriasis, two ZNF750 haplotypes showed a significant association. We also observed a nominal association between rare variants in the 5' regulatory region of ZNF750 and psoriasis. Collectively, these rare 5' regulatory variants were seen in 10 of $716(1.4 \%)$ of the psoriasis population surveyed. Functional assays demonstrated that 4 of these variants decreased ZNF750 promoter activity in accordance with previous reports in psoriasis [12] and psoriasiform dermatitis [11]. We found that these variants did not segregate with the psoriasis phenotype within families, suggesting that they are modifier variants rather than causal for psoriasis. Further studies are warranted to determine whether these rare, non-coding variants could play an influencing role in disease expression.

\section{Acknowledgements and Funding \\ We thank Anne Bowcock for providing psoriasis samples, Olivia Lai for her technical assistance, and Dennis Oh and Susana Ortiz-Urda for providing primary keratinocytes for culture. WL was supported by grants from the Dermatology Foundation and National Institute of Arthritis, Musculoskeletal and Skin Diseases (K08AR057763). IC and OSB were supported in part by a grant from the Israel Science Foundation (ISF). The funding sources had no role in the study design, collection, analysis, and interpretation of data, in the writing of the manuscript, or in the decision to submit the manuscript for publication.}

\section{Author details \\ ${ }^{1}$ Department of Bioengineering and Therapeutic Sciences, University of California, San Francisco, California, USA. ${ }^{2}$ Institute for Human Genetics, University of California, San Francisco, California, USA. ${ }^{3}$ The Morris Kahn Laboratory of Human Genetics, NIBN, Ben-Gurion University, Beer-Sheva, Israel. ${ }^{4}$ Department of Dermatology, University of California, San Francisco, California, USA. ${ }^{5}$ Cardiovascular Research Institute, University of California, San Francisco, California, USA.}

\section{Authors' contributions}

RYB conceived the study, performed and supervised the functional work, and helped write the manuscript. GH performed the luciferase assays and helped write the manuscript. IC performed functional characterization of the promoter region. AP performed the sequencing. $\mathrm{HC}$ performed statistical analysis. EL performed statistical analysis. PK provided logistical and financial support. OSB supervised some of the functional work and provided financial support. WL designed the study, contributed cases and controls, supervised the sequencing and analysis, helped write the manuscript, and provided financial support. All authors have read and approved the final manuscript.

\section{Competing interests}

The authors declare that they have no competing interests.

Received: 8 June 2011 Accepted: 20 December 2011

Published: 20 December 2011

\section{References}

1. Kurd SK, Gelfand JM: The prevalence of previously diagnosed and undiagnosed psoriasis in US adults: results from NHANES 2003-2004. J Am Acad Dermatol 2009, 60(2):218-224.

2. Capon F, Bijlmakers MJ, Wolf N, Quaranta M, Huffmeier U, Allen $M_{\text {, }}$ Timms K, Abkevich V, Gutin A, Smith R, Warren RB, Young HS, Worthington J, Burden AD, Griffiths CE, Hayday A, Nestle FO, Reis A, Lanchbury J, Barker JN, Trembath RC: Identification of ZNF313/RNF114 as a novel psoriasis susceptibility gene. Hum Mol Genet 2008, 17(13):1938-1945.

3. de Cid R, Riveira-Munoz E, Zeeuwen PL, Robarge J, Liao W, Dannhauser EN, Giardina E, Stuart PE, Nair R, Helms C, Escaramis G, Ballana E, MartinEzquerra G, den Heijer M, Kamsteeg M, Joosten I, Eichler EE, Lazaro C, Pujol RM, Armengol L, Abecasis G, Elder JT, Novelli G, Armour JA, Kwok PY, Bowcock A, Schalkwijk J, Estivill X: Deletion of the late cornified envelope LCE3B and LCE3C genes as a susceptibility factor for psoriasis. Nat Genet 2009, 41(2):211-215

4. Ellinghaus E, Ellinghaus D, Stuart PE, Nair RP, Debrus S, Raelson JV, Belouchi M, Fournier H, Reinhard C, Ding J, Li Y, Tejasvi T, Gudjonsson J, Stoll SW, Voorhees JJ, Lambert S, Weidinger S, Eberlein B, Kunz M, Rahman P, Gladman DD, Gieger C, Wichmann HE, Karlsen TH, Mayr G, Albrecht M, Kabelitz D, Mrowietz U, Abecasis GR, Elder JT, Schreiber S, Weichenthal M, Franke A: Genome-wide association study identifies a psoriasis susceptibility locus at TRAF3IP2. Nat Genet 2010, 42(11):991-995.

5. Huffmeier U, Uebe S, Ekici AB, Bowes J, Giardina E, Korendowych E, Juneblad K, Apel M, McManus R, Ho P, Bruce IN, Ryan AW, Behrens F, Lascorz J, Bohm B, Traupe H, Lohmann J, Gieger C, Wichmann HE, Herold C, Steffens M, Klareskog L, Wienker TF, Fitzgerald O, Alenius GM, McHugh NJ, Novelli G, Burkhardt H, Barton A, Reis A: Common variants at TRAF3IP2 are associated with susceptibility to psoriatic arthritis and psoriasis. Nat Genet 2010, 42(11):996-999.

6. Liu Y, Helms C, Liao W, Zaba LC, Duan S, Gardner J, Wise C, Miner A, Malloy MJ, Pullinger CR, Kane JP, Saccone S, Worthington J, Bruce I, Kwok PY, Menter A, Krueger J, Barton A, Saccone NL, Bowcock AM: A genome-wide association study of psoriasis and psoriatic arthritis identifies new disease loci. PLoS Genet 2008, 4(3):e1000041.

7. Nair RP, Duffin KC, Helms C, Ding J, Stuart PE, Goldgar D, Gudjonsson JE, Li Y, Tejasvi T, Feng BJ, Ruether A, Schreiber S, Weichenthal M, Gladman D, Rahman P, Schrodi SJ, Prahalad S, Guthery SL, Fischer J, Liao W, Kwok PY, Menter A, Lathrop GM, Wise CA, Begovich AB, Voorhees JJ, Elder JT, Krueger GG, Bowcock AM, Abecasis GR: Genome-wide scan reveals association of psoriasis with IL-23 and NF-kappaB pathways. Nat Genet 2009, 41(2):199-204.

8. Strange A, Capon F, Spencer CC, Knight J, Weale ME, Allen MH, Barton A, Band G, Bellenguez C, Bergboer JG, Blackwell JM, Bramon E, Bumpstead SJ, Casas JP, Cork MJ, Corvin A, Deloukas P, Dilthey A, Duncanson A, Edkins S, Estivill X, Fitzgerald O, Freeman C, Giardina E, Gray E, Hofer A, Huffmeier U, Hunt SE, Irvine AD, Jankowski J, Kirby B, Langford C, Lascorz J, Leman J, Leslie S, Mallbris L, Markus HS, Mathew CG, McLean WH, McManus R, Mossner R, Moutsianas L, Naluai AT, Nestle FO, Novelli G, Onoufriadis A, Palmer CN, Perricone C, Pirinen M, Plomin R, Potter SC, Pujol RM, Rautanen A, Riveira-Munoz E, Ryan AW, Salmhofer W, Samuelsson L, Sawcer SJ, Schalkwijk J, Smith CH, Stahle M, Su Z, Tazi-Ahnini R, Traupe H, Viswanathan AC, Warren RB, Weger W, Wolk K, Wood N, Worthington J, Young HS, Zeeuwen PL, Hayday A, Burden AD, Griffiths CE, Kere J, Reis A, McVean G, Evans DM, Brown MA, Barker JN, Peltonen L, Donnelly P, Trembath RC: A genome-wide association study identifies new psoriasis 
susceptibility loci and an interaction between HLA-C and ERAP1. Nat Genet 2010, 42(11):985-990.

9. Stuart PE, Nair RP, Ellinghaus E, Ding J, Tejasvi T, Gudjonsson JE, Li Y, Weidinger S, Eberlein B, Gieger C, Wichmann HE, Kunz M, Ike R, Krueger GG, Bowcock AM, Mrowietz U, Lim HW, Voorhees JJ, Abecasis GR,

Weichenthal M, Franke A, Rahman P, Gladman DD, Elder JT: Genome-wide association analysis identifies three psoriasis susceptibility loci. Nat Genet 2010, 42(11):1000-1004.

10. Chen H, Poon A, Yeung C, Helms C, Pons J, Bowcock AM, Kwok PY, Liao W: A genetic risk score combining ten psoriasis risk loci improves disease prediction. PLoS One 2011, 6(4):e19454.

11. Birnbaum RY, Zvulunov A, Hallel-Halevy D, Cagnano E, Finer G, Ofir R, Geiger D, Silberstein E, Feferman Y, Birk OS: Seborrhea-like dermatitis with psoriasiform elements caused by a mutation in ZNF750, encoding a putative $\mathrm{C} 2 \mathrm{H} 2$ zinc finger protein. Nat Genet 2006, 38(7):749-751.

12. Yang CF, Hwu WL, Yang LC, Chung WH, Chien YH, Hung CF, Chen HC, Tsai PJ, Fann CS, Liao F, Chen YT: A promoter sequence variant of ZNF750 is linked with familial psoriasis. J Invest Dermatol 2008, 128(7):1662-1668.

13. Madsen BE, Browning SR: A groupwise association test for rare mutations using a weighted sum statistic. PLoS Genet 2009, 5(2):e1000384.

14. Bansal V, Libiger O, Torkamani A, Schork NJ: Statistical analysis strategies for association studies involving rare variants. Nat Rev Genet 2010, 11(11):773-785.

15. Kimura K, Wakamatsu A, Suzuki Y, Ota T, Nishikawa T, Yamashita R, Yamamoto J, Sekine M, Tsuritani K, Wakaguri H, Ishii S, Sugiyama T, Saito K, Isono Y, Irie R, Kushida N, Yoneyama T, Otsuka R, Kanda K, Yokoi T, Kondo H, Wagatsuma M, Murakawa K, Ishida S, Ishibashi T, Takahashi-Fujii A, Tanase T, Nagai K, Kikuchi H, Nakai K, Isogai T, Sugano S: Diversification of transcriptional modulation: large-scale identification and characterization of putative alternative promoters of human genes. Genome Res 2006, 16(1):55-65.

16. Enlund F, Samuelsson L, Enerback C, Inerot A, Wahlstrom J, Yhr M, Torinsson A, Martinsson T, Swanbeck G: Analysis of three suggested psoriasis susceptibility loci in a large Swedish set of families: confirmation of linkage to chromosome $6 p$ (HLA region), and to $17 q$, but not to 4q. Hum Hered 1999, 49(1):2-8.

17. Nair RP, Henseler T, Jenisch S, Stuart P, Bichakjian CK, Lenk W, Westphal E, Guo SW, Christophers E, Voorhees JJ, Elder JT: Evidence for two psoriasis susceptibility loci (HLA and 17q) and two novel candidate regions (16q and 20p) by genome-wide scan. Hum Mol Genet 1997, 6(8):1349-1356.

18. Tomfohrde J, Silverman A, Barnes R, Fernandez-Vina MA, Young M, Lory D, Morris L, Wuepper KD, Stastny P, Menter A, et al: Gene for familial psoriasis susceptibility mapped to the distal end of human chromosome 17q. Science 1994, 264(5162):1141-1145.

19. Zheng J, Jin S, Shi R: Confirmation of PSORS psoriasis susceptibility loci in a Chinese population. Arch Dermatol Res 2003, 295(1):14-18.

20. Ionita-Laza I, Lange CNML: Estimating the number of unseen variants in the human genome. Proc Natl Acad Sci USA 2009, 106(13):5008-5013.

21. Neale BM, Rivas MA, Voight BF, Altshuler D, Devlin B, Orho-Melander M, Kathiresan S, Purcell SM, Roeder K, Daly MJ: Testing for an unusual distribution of rare variants. PLoS Genet 2011, 7(3):e1001322.

22. Elder JT, Nair RP, Guo SW, Henseler T, Christophers E, Voorhees JJ: The genetics of psoriasis. Arch Dermatol 1994, 130(2):216-224.

23. Albanesi C, De Pita O, Girolomoni G: Resident skin cells in psoriasis: a special look at the pathogenetic functions of keratinocytes. Clin Dermatol 2007, 25(6):581-588.

24. Gupta AK, Madzia SE, Batra R: Etiology and management of Seborrheic dermatitis. Dermatology 2004, 208(2):89-93.

\section{Pre-publication history}

The pre-publication history for this paper can be accessed here: http://www.biomedcentral.com/1471-2350/12/167/prepub

\section{doi:10.1186/1471-2350-12-167}

Cite this article as: Birnbaum et al: Association analysis identifies ZNF750 regulatory variants in psoriasis. BMC Medical Genetics 2011 12:167.

\section{Submit your next manuscript to BioMed Central and take full advantage of:}

- Convenient online submission

- Thorough peer review

- No space constraints or color figure charges

- Immediate publication on acceptance

- Inclusion in PubMed, CAS, Scopus and Google Scholar

- Research which is freely available for redistribution

Submit your manuscript at www.biomedcentral.com/submit
Biomed Central 